\title{
Thermodynamics of the two-dimensional Hubbard model based on the exact two-body S-matrix
}

\author{
André LeClair \\ Newman Laboratory, Cornell University, Ithaca, NY and \\ Centro Brasileiro de Pesquisas Físicas, Rio de Janeiro
}

\begin{abstract}
A new analytic treatment of the two-dimensional Hubbard model at finite temperature and chemical potential is presented. A next nearest neighbor hopping term of strength $t^{\prime}$ is included. This analysis is based upon a formulation of the statistical mechanics of particles in terms of the S-matrix. We show that for $U / t$ large enough, a region of attractive interactions exists near the Fermi surface due to multi-loop quantum corrections. For $t^{\prime}=-0.3$, these attractive interactions exist for $U / t>6.4$. Our analysis suggests that superconductivity may not exist for $t^{\prime}=0$. Based on the existence of solutions of the integral equation for the pseudo-energy, we provide evidence for a phase transition and estimate $T_{c} / t \approx 0.02$ for $U / t=7.5$ and $t^{\prime} / t=-0.3$ at hole doping 0.15 .
\end{abstract}




\section{INTRODUCTION}

The Hubbard model in two spatial dimensions plays a central role in the modern theory of strongly correlated electrons. Since it is believed to be a good microscopic model for the underlying physics of high $T_{c}$ superconductivity (HTSC) [1], it has been studied extensively over the past two decades. A partial list of publications on the thermodynamics of the Hubbard model is [2 10]. Despite this effort, many of its important properties are not currently well-understood, and it remains to be established definitively that it possesses all of the main features of HTSC. For instance, the precise mechanism that leads to attractive d-wave pairing, which must arise from the purely electronic Coulomb repulsion, is still not well understood. Analytic methods to date are rather limited since the model is in the strong coupling regime. Lattice Monte-Carlo methods on the other hand are limited to small lattices and suffer from the fermion sign problem, especially at non-zero doping and sufficiently low temperatures. For these reasons, any new analytic methods, though approximate, may shed new light on the problem.

In this work we present an analytic approach to the thermodynamics of particles at finite density and temperature based on the reformulation of the statistical mechanics of particles in terms of the zero temperature and density particle-particle S-matrix developed in [11, 12]. As explained there, the potential advantage of this method is that, unlike the usual diagramatic Matsubara approach to finite temperature field theory, it disentangles the zero temperature dynamics from the quantum statistical sums. Some remarks clarifying the nature of this formalism, and the approximations made, are called for. The approach was modeled after the thermodynamic Bethe ansatz[13], which is exact for integrable models in one spatial dimension since the Nbody S-matrix factorizes into 2-body S-matrices, Our method indeed reduces to the thermodynamic Bethe ansatz in the two-body scattering approximation, as shown 
in [12] for the interacting 1d Bose gas. The main approximation we make is that we consider only many-body processes that involve arbitrary numbers of primitive binary collisions. I.e. we neglect processes that in some sense involve 3 or more particles colliding simultaneously, which is not the same as ignoring the many-body aspect of the problem altogether. In this non-relativistic context, it is well-known that the two-body S-matrix can be calculated exactly, thus in some regards the method is non-perturbative. Although this is not a fully controlled approximation, it has been demonstrated to give reliable results for other strong-coupling problems, in particular the critical point of the $2 \mathrm{~d}$ bosonic gas and more importantly to Bose and Fermi gases in the 3d scale-invariant unitary limit[12, 14]. For instance for the unitary Fermi gas on the BEC side of the crossover, the critical temperature calculated is consistent with Monte-Carlo methods, and the ratio of the viscosity to entropy density agrees very well with the most recent experimental data[15]. It should be pointed out that the exact Bethe-ansatz solution of the 1d Hubbard model exhibits two additional holon excitations [16], which can be inferred from poles in the S-matrix of the fundamental fermions; we have no evidence for such excitations in the 2-dimensional case, thus it is unclear whether a meaningful comparison with the 1d case can be made.

Our conventions for the Hubbard model are described in the next section. We include a next nearest neighbor hopping term of strength $t^{\prime}$, since it is known to be non-zero in the cuprates; as we will show, its effects are important. In section III, the effective momentum dependent coupling, which is the kernel $G\left(\mathbf{k}_{1}, \mathbf{k}_{2}\right)$ constructed from the logarithm of the 2-body S-matrix in the integral equation satisfied by the pseudo-energy, is analyzed. We show that there exists a band of attractive interactions near the half-filled Fermi surface for $U / t$ large enough, and $t^{\prime}$ plays an important role in determining this property. We emphasize that no approximations are made in section III, since, as stated above, the 2-body S-matrix can be calculated 
exactly, and this attractive region exists regardless of the subsequent approximations we make in studying the thermodynamics. This attractive mechanism, which arises from quantum loop corrections, appears to be different than other mechanisms discussed in this context, such as those based on spin fluctuations or the resonating valence bond picture. It is thus important to investigate the consequences of these attractive interactions and how they might be connected to HTSC, and this paper is a first step in this direction. In section IV the S-matrix based formalism we utilize for calculating thermodynamic properties is reviewed and specialized to the Hubbard gas. In section $\mathrm{V}$ the free energy is analyzed, and we present some evidence for phase transitions.

\section{HUBBARD MODEL CONVENTIONS}

The Hubbard model describes fermionic particles with spin, hopping between the sites of a square lattice, subject to strong local coulombic repulsion. The lattice hamiltonian is

$$
H=-t \sum_{<i, j>, \alpha=\uparrow, \downarrow}\left(c_{\mathbf{r}_{i}, \alpha}^{\dagger} c_{\mathbf{r}_{j}, \alpha}\right)-t^{\prime} \sum_{<i, j>\prime, \alpha=\uparrow, \downarrow}\left(c_{\mathbf{r}_{i}, \alpha}^{\dagger} c_{\mathbf{r}_{j}, \alpha}\right)+U \sum_{\mathbf{r}} n_{\mathbf{r} \uparrow} n_{\mathbf{r} \downarrow}
$$

where $\mathbf{r}_{i, j}, \mathbf{r}$ are sites of the lattice, $\left\langle i, j>\right.$ denotes nearest neighbors, $n=c^{\dagger} c$ are

densities, and $c^{\dagger}, c$ satisfy canonical anti-commutation relations. For both cuprates LSCO and BSCO, $U / t \approx 13$. We have also included a next to nearest neighbor hopping term $t^{\prime}$, since it is not difficult to incorporate into the formalism, and it is known to be non-zero for high $T_{c}$ materials. As we will see, it can play a significant role. For LSCO and BSCO, $t^{\prime} / t$ approximately equals -0.1 and -0.3 respectively; in our analysis below we set $t^{\prime} / t=-0.3$. 
We introduce the two fields $\psi_{\uparrow, \downarrow}$ and the action

$$
S=\int d^{2} \mathbf{r} d t\left(\sum_{\alpha=\uparrow, \downarrow} i \psi_{\alpha}^{\dagger} \partial_{t} \psi_{\alpha}-\mathcal{H}\right)
$$

where $\mathcal{H}$ is the hamiltonian density. The field has the following expansion characteristic of a non-relativistic theory since it only involves annihilation operators,

$$
\psi_{\alpha}(\mathbf{r})=\int \frac{d^{2} \mathbf{k}}{2 \pi} c_{\mathbf{k}, \alpha} e^{i \mathbf{k} \cdot \mathbf{r}}
$$

and satisfies

$$
\left\{\psi_{\alpha}(\mathbf{r}), \psi_{\alpha^{\prime}}^{\dagger}\left(\mathbf{r}^{\prime}\right)\right\}=\delta\left(\mathbf{r}-\mathbf{r}^{\prime}\right) \delta_{\alpha, \alpha^{\prime}}
$$

Since we have represented sums over lattice sites $\mathbf{r}$ as $\int d^{2} \mathbf{r} / a^{2}$, where $a$ is the lattice spacing, $c_{\mathbf{r}}=a \psi(\mathbf{r})$. The free part of the hamiltonian is then

$$
H_{\text {free }}=\int d^{2} \mathbf{k} \omega_{\mathbf{k}} \sum_{\alpha} c_{\mathbf{k}, \alpha}^{\dagger} c_{\mathbf{k}, \alpha}
$$

with the 1-particle energy

$$
\omega_{\mathbf{k}}=-2 t\left(\cos \left(k_{x} a\right)+\cos \left(k_{y} a\right)\right)-4 t^{\prime} \cos \left(k_{x} a\right) \cos \left(k_{y} a\right)
$$

where $t$ taken to be positive. In the sequel it is implicit that $\mathbf{k}$ is restricted to the first Brillouin zone, $-\pi / a \leq k_{x, y} \leq \pi / a$

The interaction part of the hamiltonian is local, and becomes a continuum integral:

$$
H_{\mathrm{int}}=\frac{u}{2} \int d^{2} \mathbf{r} \quad \psi_{\uparrow}^{\dagger} \psi_{\uparrow} \psi_{\downarrow}^{\dagger} \psi_{\downarrow}
$$

where $u=2 U a^{2}$. The model is now viewed as a quantum fermionic gas, where the only effect of the lattice is in the free particle energies $\omega_{\mathbf{k}}$.

The field $\psi$ has dimensions of inverse length, and the coupling $u$ units of energy • length $^{2}$. In the sequel we will scale out the dependence on $t$ and the lattice spacing $a$, and physical quantities will then depend on the dimensionless coupling

$$
g=\frac{u}{a^{2} t}=\frac{2 U}{t}
$$

Positive $g$ corresponds to repulsive interactions. 


\section{THE EFFECTIVE MOMENTUM DEPENDENT COUPLING AND THE POSSIBLE ORIGIN OF ATTRACTIVE INTERACTIONS}

In the finite temperature formalism developed in [11, 12], the occupation numbers $f$ are parameterized in terms of a pseudo-energy $\varepsilon(\mathbf{k})$ in the same manner as for a free theory: $f=1 /\left(e^{\beta \varepsilon}+1\right)$. In the approximation that only the many-body processes built out of primitive 2-body collisions are retained, the pseudo-energy satisfies an integral equation based on a kernel $G\left(\mathbf{k}_{1}, \mathbf{k}_{2}\right)$ which is related to the logarithm of the 2-particle S-matrix. This approach to the thermodynamics will be reviewed in the next section. The final result derived in [12] involves only the particle-particle S-matrix at zero temperature and density, which can be calculated exactly. The temperature and density enter the formalism in the integral equation for the pseudoenergy, thus this formalism does not require particle-particle or particle-hole Green's functions at finite temperature and chemical potential. In this section we study the main features of the kernel and demonstrate that there are regions of the Brillouin zone where the interactions are effectively attractive, even though the bare model has repulsive interactions. We we wish to emphasize that no approximations are made in obtaining the results presented in this section, which essentially amount to quantum corrections to scattering, and some conclusions are independent of the thermodynamics studied in subsequent sections.

\section{A. Structure of the kernel}

As described in [12], the kernel has the following structure:

$$
G\left(\mathbf{k}_{1}, \mathbf{k}_{2}\right)=-\frac{i}{2 \mathcal{I}} \log (1+2 i \mathcal{I} \mathcal{M})
$$


where $\mathcal{M}$ is the 2-body scattering amplitude. (We are suppressing the momentum dependence.) $\mathcal{I}$ represents the available phase space for two-body scattering:

$$
\mathcal{I}=\frac{1}{4 \pi} \int d^{2} \mathbf{p} \delta\left(E-\omega_{\mathbf{p}}-\omega_{\mathbf{K}-\mathbf{p}}\right)
$$

where $E$ and $\mathbf{K}$ are the total energy and momentum of the two incoming particles with momenta $\mathbf{k}_{1}$ and $\mathbf{k}_{2}$ :

$$
E=\omega_{\mathbf{k}_{1}}+\omega_{\mathbf{k}_{2}}, \quad \mathbf{K}=\mathbf{k}_{1}+\mathbf{k}_{2}
$$

All energy and temperature scales, $E, T$, the chemical potential $\mu$, and $t^{\prime}$, will be expressed in units of the hopping parameter $t$. We thus scale $t$ out of $\omega_{\mathbf{k}}$ so that henceforth $\omega_{\mathbf{k}}$ equals (6) divided by $t$. We will also rescale $\mathbf{k}$ by $1 / a$ so that $-\pi \leq k_{x, y} \leq \pi$. Since $\mathcal{I}$ is proportional to $1 / t, G \propto t$, and henceforth $G$ will represent $G / t$, which is dimensionless after scaling out factors of $a$ also. The kernel $G$ then depends only on the dimensionless coupling $g$ defined in eq. (8), and the momenta.

The scattering amplitude can be computed by summing multi-loop ladder diagrams[12], which factorize into 1-loop integrals in this non-relativistic context. This leads to

$$
\mathcal{M}=\frac{-g / 2}{1+i g L / 2}
$$

where $g$ is the coupling defined in eq. (8). $L$ is a 1-loop integral:

$$
\begin{aligned}
L & =\int_{-\infty}^{\infty} \frac{d \omega}{2 \pi} \int \frac{d^{2} p}{(2 \pi)^{2}}\left(\frac{i}{\omega-\omega_{\mathbf{p}}+i \epsilon}\right)\left(\frac{i}{E-\omega-\omega_{\mathbf{K}-\mathbf{p}}+i \epsilon}\right) \\
& =i \int \frac{d^{2} \mathbf{p}}{(2 \pi)^{2}} \frac{1}{E-\omega_{\mathbf{p}}-\omega_{\mathbf{K}-\mathbf{p}}+2 i \epsilon}
\end{aligned}
$$

where $\epsilon$ is small and positive. In the numerical analysis below we set $\epsilon=0.001$. Using $\operatorname{Im}(x+i \epsilon)^{-1}=-\pi \delta(x)$, one sees that

$$
L=\mathcal{I}+i \gamma
$$


where $\mathcal{I}$ is the phase space factor in eq. (10) and is real and positive, and $\gamma$ is its imaginary part. Putting all of this together one has

$$
G=-\frac{i}{2 \mathcal{I}} \log \left(\frac{1 / g_{R}-i \mathcal{I} / 2}{1 / g_{R}+i \mathcal{I} / 2}\right)
$$

The imaginary part of the loop integral renormalizes the coupling $g$

$$
g_{R}=\frac{g}{1-g \gamma / 2}
$$

Note that the manner in which $g_{R}$ enters the kernel leads to a well-defined large coupling limit; this was exploited for unitary quantum gases in [14] where $g_{R}$ is proportional to the scattering length which goes to $\pm \infty$ in the unitary limit.

The argument of the log in eq. (15) can be identified as the 2-body S-matrix, which is unitary, i.e. $S^{*} S=1$. It should be emphasized that this is the exact two-body S-matrix, and this is possible because the model is non-relativistic. More specifically, the fields $\psi_{\uparrow, \downarrow}$ only involve annihilation operators, in contrast to relativistic theories which are expanded in both creation and annihilation operators. Let us elaborate on this important point, which is well-known in other contexts, such as non-relativistic quantum gases, but often not completely explained. Represent the interaction vertex with two incoming arrows for the annihilation operator fields $\psi_{\uparrow, \downarrow}$ and two outgoing arrows for the creation fields $\psi_{\uparrow, \downarrow}^{\dagger}$. Consider for simplicity the 1-loop contributions. Diagrams with a closed loop, i.e. with arrows circulating in the same directions, such as the second diagram in Figure 1, are zero because the integration over energy $\omega$ inside the loop has poles in the integrand that are either both in the upper or lower half-plane, so that the contour can be closed at infinity without picking up residues. (This is only true because our formalism only involves the particle-particle S-matrix at zero temperature and density.) In other words, there is no "crossingsymmetry" as in relativistic theories, where there are three non-zero 1-loop diagrams, with different momentum dependence, which are crossed versions of each other. The 
non-zero multi-loop diagrams are only of the "ladder type", which factorize, and this was implicitly used in [12]. There is actually no fermionic minus sign associated with each loop since the arrows do not form a closed loop. Since this S-matrix is exact to all orders in $g$, the thermodynamic formalism we will use embodies some nonperturbative aspects of the problem, although it still represents an approximation to the thermodynamics, as explained in more detail in the next section.
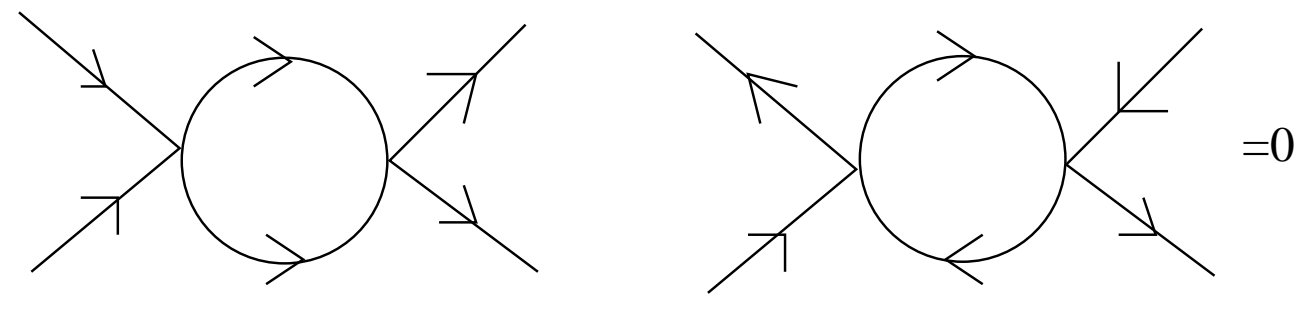

FIG. 1: One-loop contributions to the S-matrix. Only the diagram to the left is non-zero.

\section{B. Origin of attractive interactions}

The kernel $G$ by construction is real. For small coupling $g, G$ is independent of momentum and equal to $-g / 2$. Thus $G$ may be viewed as an effective, momentumdependent coupling constant, and provides valuable information on the effective 2body interactions at zero temperature. When $G$ is negative the interactions are effectively repulsive, otherwise they are attractive. The important point is that the renormalization of $g$ to $g_{R}$ can in fact change the sign of $g_{R}$, which changes the sign of $G$ due to the branch cut in the logarithm. To demonstrate how this can happen, we first perform the $p_{y}$ integral in $L$. The result is

$L=\frac{i}{2 \pi^{2}} \int_{-\pi}^{\pi} d p_{x} \frac{1}{\sqrt{B^{2}+C^{2}-D^{2}}}\left[\log \left(\frac{(C-D)}{\sqrt{B^{2}+C^{2}-D^{2}}}\right)-\log \left(\frac{(D-C)}{\sqrt{B^{2}+C^{2}-D^{2}}}\right)\right]$ 
where

$$
\begin{aligned}
& B=2 \sin K_{y}+4 t^{\prime} \cos \left(K_{x}-p_{x}\right) \sin K_{y} \\
& C=2+2 \cos K_{y}+4 t^{\prime} \cos p_{x}+4 t^{\prime} \cos \left(K_{x}-p_{x}\right) \cos K_{y} \\
& D=E+2 \cos p_{x}+2 \cos \left(K_{x}-p_{x}\right)+2 i \epsilon
\end{aligned}
$$

(Recall $t^{\prime}$ represents $t^{\prime} / t$.) The sum of the logarithms in the above formula is simply $\log (-1)= \pm i \pi$; however expressing the integral in this fashion ensures one is on the proper branch. The above formula proved to be very useful for the numerical evaluation of the kernel.

From the expression for the renormalized coupling $g_{R}$, eq. (16), one sees that $g_{R}$ can become negative if $\gamma$ is positive and $g$ large enough, $g>2 / \gamma$. Let $k_{1}, k_{2}$ be in the center of mass frame, $\mathbf{k}_{1}=-\mathbf{k}_{2}=\mathbf{k}$, so that $\mathbf{K}=0$, and $L$ only depends on the total energy $E$. The loop integral (17) can be expressed in terms of elliptic functions. To regulate the integral, we let the upper limit of the $p_{x}$ integral be $\pi-\kappa$, and then let $\kappa \rightarrow 0$. The loop integral $L \propto F(i \log (4 a / \kappa), b)$ where $F$ is the elliptic integral of the first kind, with $a=\sqrt{\left(E-8+8 t^{\prime}\right) /\left(E-8 t^{\prime}\right)}$ and $b=\left(E-8 t^{\prime}\right)^{2} /\left(\left(E+8 t^{\prime}\right)^{2}-64\right)$. As $\kappa \rightarrow 0$, we use $\lim _{x \rightarrow \infty} F(i x, b)=i K(1-b)$, where $K$ is the complete elliptic integral of the first kind. The final result is

$$
L_{\mathbf{K}=0}=\frac{2}{\pi}\left(\frac{E-8 t^{\prime}}{\left(E+8+8 t^{\prime}\right)\left(E(8-E)+64 t^{\prime}\left(t^{\prime}-1\right)\right)}\right)^{1 / 2} K\left(\frac{32\left(E t^{\prime}-2\right)}{\left(E+8 t^{\prime}\right)^{2}-64}\right)
$$

with $E \rightarrow E+2 i \epsilon$. The flip in sign is a result of the combination of logarithms in eq. (17).

The imaginary part of $L$, i.e. $\gamma$, is plotted in Figure 2 for $t^{\prime}=-0.3$. Since $\gamma$ is positive for large enough $E$, one reaches the remarkable conclusion that for $g$ large enough, the effective interactions can become attractive. One can estimate this threshold for $g$ as $2 / \gamma_{\max }$, where $\gamma_{\max }$ is the maximum value of $\gamma$ which occurs 
where $\gamma$ flips sign. This value of $\gamma_{\max }$ can be obtained using the formula (19), and is $\gamma_{\max } \approx 0.156$ for $t^{\prime}=-0.3$. This minimal threshold in $g$ should be contrasted with the Cooper instability, which leads to superconductivity for arbitarily weak coupling. This fact is a consequence of a logarithmic divergence in the analogous loop integrals, which leads a gap proportional to $e^{-1 / u}$ for some appropriate coupling $u$. In the present context, the attractive interactions arising from the above change of sign do not involve an analogous logarithmic divergence, hence the threshold.

In summary, we have shown that there are effectively attractive interactions above a threshold in $U / t$, e.g. for $t^{\prime}=-0.3$, attractive interactions exist for $g>12.8$, i.e. $U / t>6.4$. We repeated this analysis for other values of $t^{\prime}$ using the formula (19), and our results for the mininum value of $g$ necessary for attractive interactions are shown in the table below. A minimal threshold for superconductivity was proposed in [17], with minimal values of $U / t$ in the comparable range of $4-7$. On the other hand, the study in[18] indicates no threshold, namely, for the particular mechanism they they study, superconductivity exists for arbitrarily low $U / t$. This does not necessarily contradict our result, since the attractive mechanism we study here is essentially different, and we have not made a case yet that it is the one responsible for superconductivity.

\begin{tabular}{|c|c|}
\hline \hline$t^{\prime} / t$ & $g_{\min }$ \\
\hline \hline-0.1 & 16.7 \\
-0.2 & 14.3 \\
-0.3 & 12.8 \\
-0.4 & 10.0 \\
\hline \hline
\end{tabular}

The change in sign of the effective coupling described above is reminiscent of what is encountered in the $\mathrm{BEC} / \mathrm{BCS}$ crossover of the non-relativistic continuum three-dimensional unitary gas. The model is defined with repulsive interactions, 


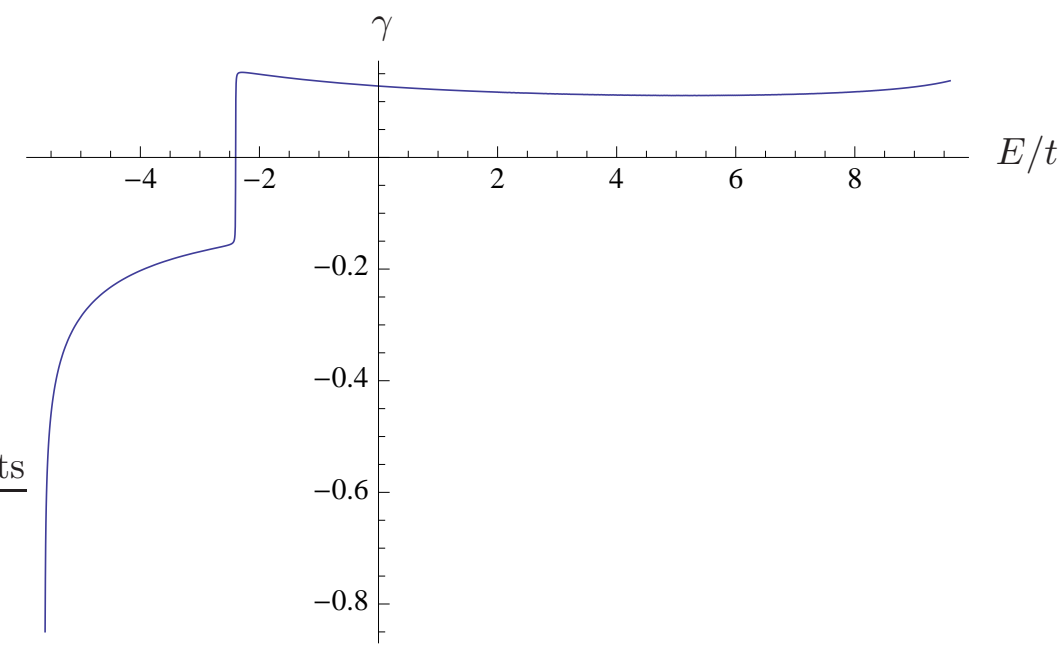

FIG. 2: The imaginary part of the loop integral $\gamma$ as a function of energy $E$ for $t^{\prime} / t=-0.3$.

i.e. positive coupling, however there is a fixed point at a negative coupling $g_{*}$, independent of momenta. The scattering length is proportional to the renormalized coupling $g_{R}=g /\left(1-g / g_{*}\right)$. Just above the fixed point, the scattering length goes to $-\infty$, whereas just below it goes to $+\infty$. The kernel $G$ for this model flips sign as one crosses the fixed point for the same reasons as above, i.e. because of the branch-cut of the logarithm[14]. Thus, the effective interactions can be repulsive or attractive, depending on which side of the fixed point one sits, even though the bare model defined by the hamiltonian had only repulsive interactions. The main difference in the Hubbard gas is that the renormalized coupling depends on the momenta, so that the interactions may become attractive in distinct regions of the Brillouin zone.

We mention that a change in sign of certain couplings under renormalization group flow was found for a 2-chain Hubbard model (2-legged ladder) in [19]. Such ladders are effectively 1 dimensional, and were mapped onto an anisotropic Gross-Neveu model, i.e. free Dirac fermions with marginal current-current interactions. Since these Gross-Neveu models are very different from those considered here, it seems 
unlikely that the change of sign described in [19] is related to the one described here, since our model is intrinsically 2-dimensional, and the phenomenon does not involve any renormalization group flow.

We now study the kernel $G\left(\mathbf{k}_{1}, \mathbf{k}_{2}\right)$ for $\mathbf{k}_{1}=-\mathbf{k}_{2}=\mathbf{k}$ and verify the above results. For these momenta, the kernel is only a function of the total energy $E$. In Figure 3. we plot $G(E)$ for the values of the coupling $g=5,13.5,14,15,20$ and $t^{\prime}=-0.3$. One observes that for the smaller $g=5$, the kernel is everywhere negative. One can verify that for $g$ large enough, the main features do not depend strongly on $g$. The most interesting region of $g$ is around $g \approx 13-15$ for $t^{\prime}=-0.3$. Comparing $g=14$ and 15, one sees that for $g=14$ the attractive band is narrower, and for $g=13$ essentially disappears. We will fix $g=15$ in our subsequent thermodynamic analysis, since this is in the interesting region and the attractive band is not too narrow.

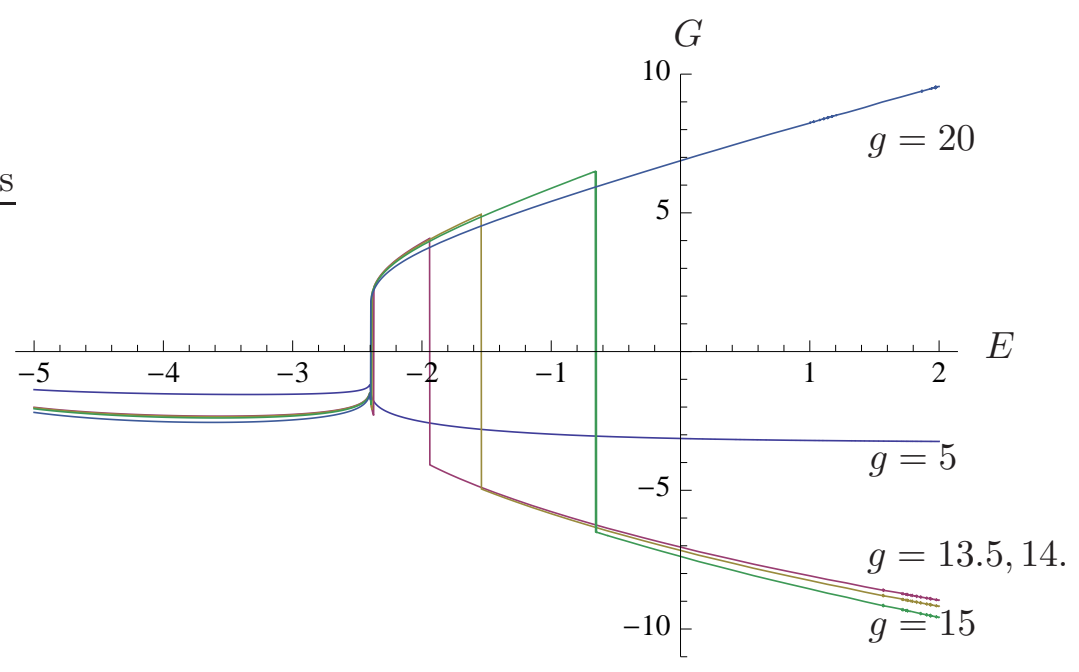

FIG. 3: The kernel $G$ as a function of total energy $E$ for $g=5,13.5,14,15,20$, and $t^{\prime} / t=-0.3$ (Color figures on-line.)

Figure 4 shows the kernel for $g=15$ for $t^{\prime} / t=0,-0.1,-0.3,-0.4$. One sees that the interactions are effectively attractive only around a small region centered 
at $E \approx-1.5$ for $t^{\prime} / t=-0.3$. An important feature of our analysis is that it clearly shows the importance of a non-zero $t^{\prime}$. For fixed $g$, if $\left|t^{\prime}\right|$ is too small, there is no attractive region, as is apparent in Figure 4. If superconductivity indeed arises from these attractive interactions, then this suggests that superconductivity may not exist if $t^{\prime}=0$. There is actually some experimental evidence for this, in that $T_{c}$ as a function of $t^{\prime} / t$ appears to extrapolate to zero[20]. It should be pointed out however that as one lowers $t^{\prime} / t$, attractive regions continue to exist as long as one raises the coupling $g$, as is evident in the table above for $g_{\min }$ as a function of $t^{\prime}$.

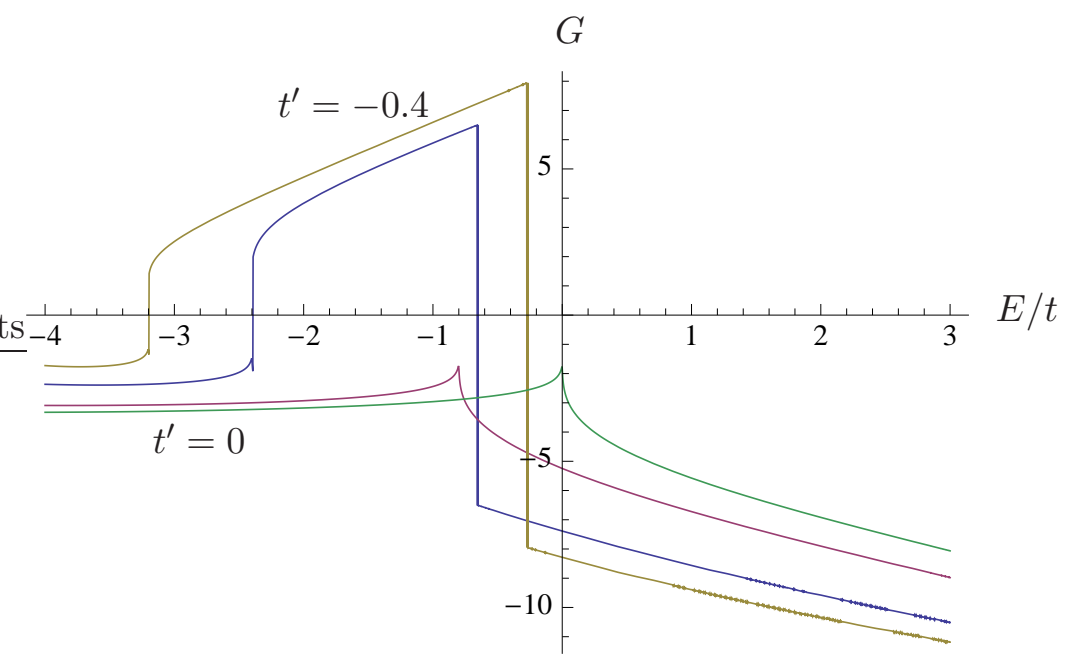

FIG. 4: The kernel $G$ as a function of total energy $E$ for $g=15$ and $t^{\prime}=0,-0.1,-0.3,-0.4$.

It is also intructive to plot $G$ for pairs of opposite momentum as a function of $k_{x}, k_{y}$ in the first Brillouin zone. This is shown in Figure 5. Again this shows that the interactions are attractive in a narrow region around half-filling. The positive regions at the corners of the Brillouin zone are due to a divergence in the loop integral which should be regularized; however since we will be studying hole doping of the half-filled state, the densities will be low enough to be far from these regions, so this regularization will be unnecessary. Finally, note that for low enough $E$, the 
interactions are always repulsive, which should imply that at high enough doping the theory should be well-approximated by a Fermi liquid.

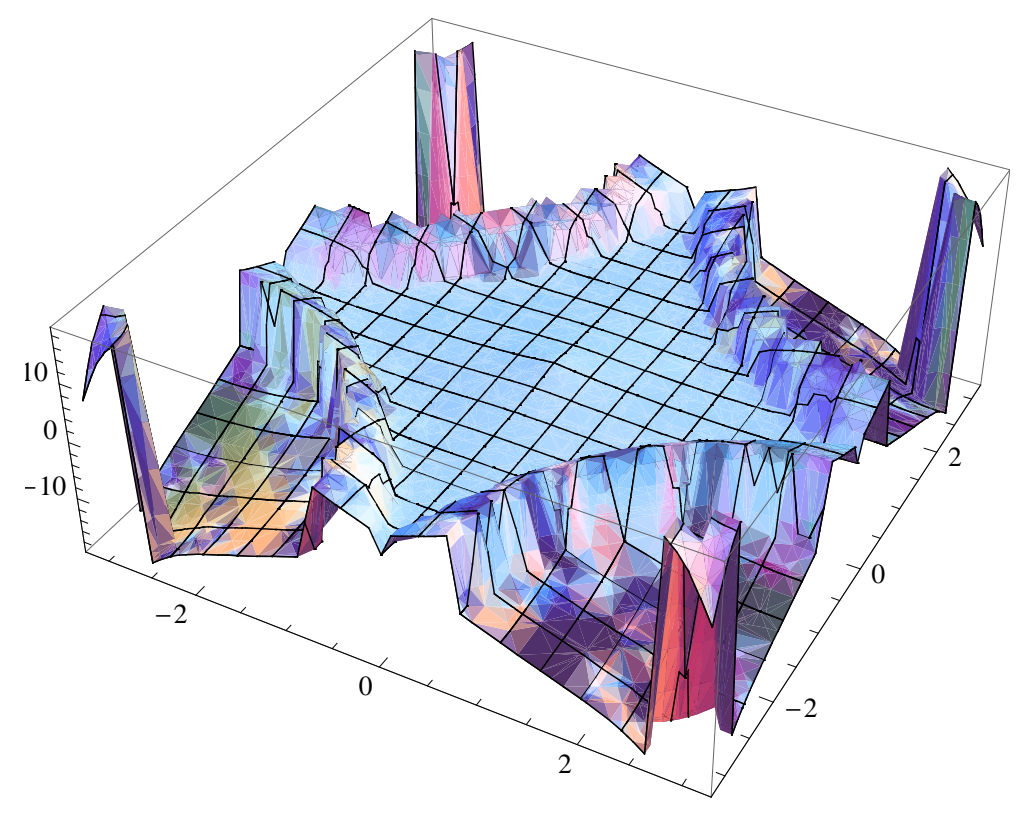

FIG. 5: The kernel $G$ for Cooper pairs in the first Brillouin zone for $g=15$ and $t^{\prime} / t=-0.3$. The horizontal axes are $-\pi<k_{x, y}<\pi$ and the vertical axis is the effective coupling $G$.

If the attractive interactions exist near the Fermi surface, then Cooper's original argument should apply: the filled Fermi sea just serves to block states and the particles can form a bound state, i.e. Cooper pairs[21]. Let us then make the hypothesis that the regions of attractive interactions described above lead to Cooper pairing. Then the following scenario emerges. The Fermi surface with interactions is calculated in section $\mathrm{V}$ based on the filling fractions $f$. In Figure 6 we plot these Fermi surfaces for various hole doping, and also display the attractive band. (See the next section for the precise definition of hole doping $h$; as defined it corresponds to the number of holes per plaquette.) These computed Fermi surfaces closely parallel experimental measurements, in that they flare out in the anti-nodal directions i .e. 
$\left(k_{x}, k_{y}\right)=(0, \pi)$ and $90^{\circ}$ rotations thereof[22]. This figure shows that the attractive regions in the anti-nodal directions play the most significant role. At low densities (high hole-doping), there are no attractive interactions within the Fermi surface, and the model should correspond to a Fermi-liquid. On the other hand, as $h$ is decreased, the Fermi surface intersects attractive regions in the anti-nodal directions. This first occurs around a hole doping $h=0.3$. If a gap forms in these directions, then this could explain the anisotropy of the gap, which is zero in the nodal $(\pi, \pi)$ directions. As the density is increased further, eventually the Fermi surface is beyond the attractive band. i.e. the attractive band is completely enclosed by the Fermi surface. At half filing, $h=0$, the attractive regions in the anti-nodal directions are just inside the Fermi surface, however the Fermi surface still intersects the attractive band in the nodal, $(\pi, \pi)$, directions.

\section{THERMODYNAMICS FROM THE S-MATRIX}

The important question that remains is whether our approach to the thermodynamics of the Hubbard model can capture the instabilities proposed at the end of the last section based on the properties of the kernel $G$. If so, this will provide a calculation of the critical temperature.

In this section we describe how to compute the free energy from a formalism that is a synthesis of the works [11, 12]. Being a synthesis, it is worthwhile reviewing the main features of the derivation, and how the construction follows from the basic ingredients in these two papers.

The starting point is a formal expression for the partition function $Z$ in terms of the S-matrix derived in [23]:

$$
Z=Z_{0}+\frac{1}{2 \pi} \int d E e^{-\beta E} \operatorname{Im} \partial_{E} \log \widehat{S}(E)
$$




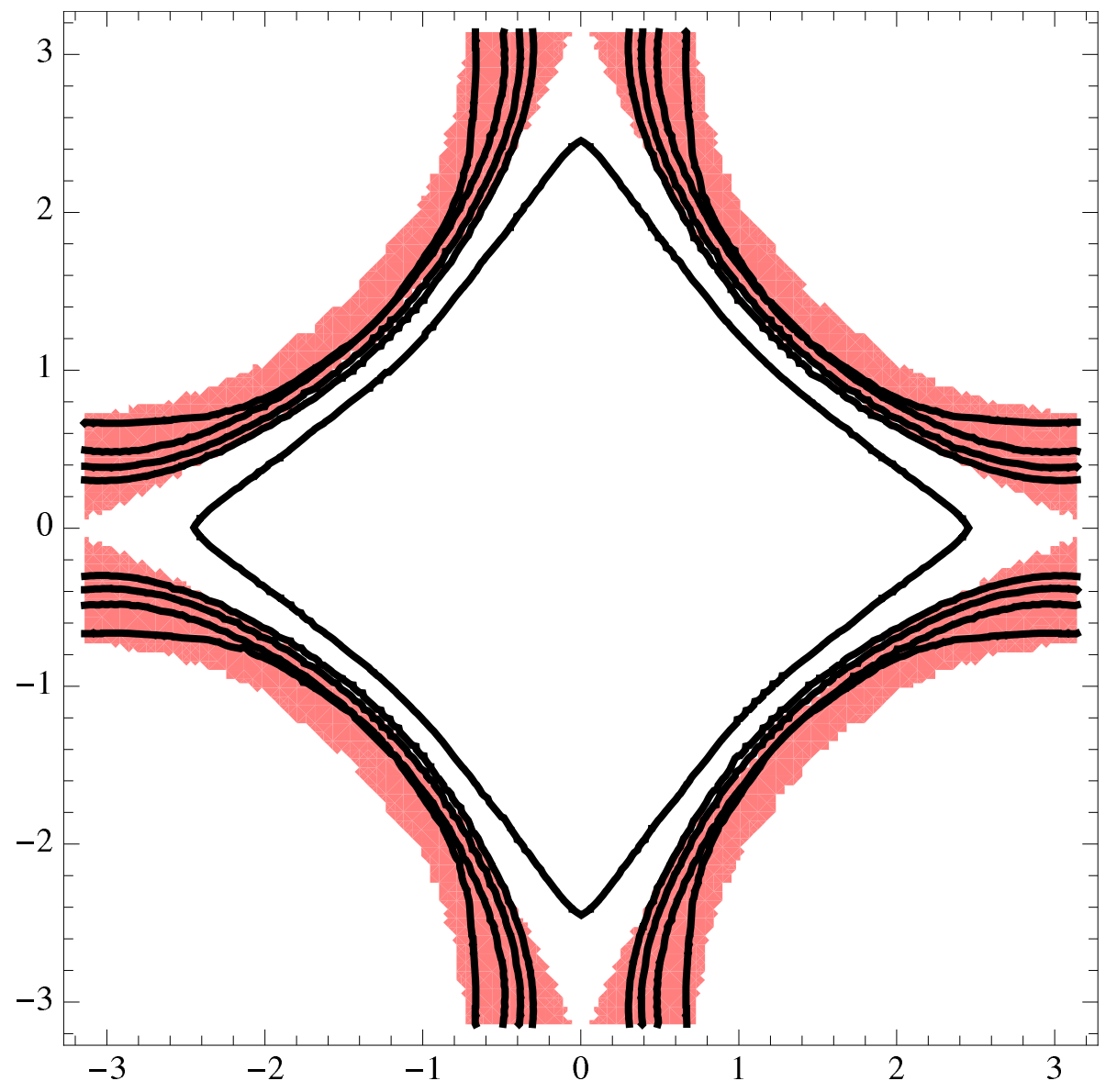

FIG. 6: Fermi surfaces for various hole doping $h=0,0.1,0.2,0.3, .04$, as computed in section V. The pink region (grey offline) is the band of attractive interactions for $g=15$. The axes are $k_{x}$ and $k_{y}$ in the first Brillouin zone, i.e. in the range $-\pi$ to $\pi$

where $\widehat{S}(E)$ is the off-shell S-matrix operator, $Z_{0}$ the free partition function, and $\beta=1 / T$. Although the above formula is simple enough, a considerable amount of additional work is needed to obtain something useful out of it. For instance the cluster decomposition property of the S-matrix is needed to show that $Z$ exponentiates to an extensive free energy. 
Consider for simplicity a single species of fermions. The basic dynamical variables are the occupation numbers $f$ which determine the density:

$$
n=\int \frac{d^{2} \mathbf{k}}{(2 \pi)^{2}} f(\mathbf{k})
$$

Using a Legendre transformation in the variables $n$ and $\mu$, where $\mu$ is the chemical potential, one can show that there exists a functional $\digamma(f)$ such that the physical free energy follows from the variational principle $\delta \digamma / \delta f=0$. This functional can be separated into a free part $\digamma_{0}$ and an interacting part $\digamma_{1}$,

$$
\digamma=\digamma_{0}+\digamma_{1}
$$

The interacting part contains contributions from $\mathrm{N}$ to $\mathrm{N}$ particle scattering for all $\mathrm{N}$. One expects the 2-particle term to be the most important and is of the form:

$$
\digamma_{1}=-\frac{1}{2} \int \frac{d^{2} \mathbf{k}}{(2 \pi)^{2}} \int \frac{d^{2} \mathbf{k}^{\prime}}{(2 \pi)^{2}} f\left(\mathbf{k}^{\prime}\right) G\left(\mathbf{k}, \mathbf{k}^{\prime}\right) f(\mathbf{k})
$$

Although $G$ is built only from the 2-body S-matrix, the formalism re-sums all manybody processes that involve arbitrary numbers of primitive binary collisions. (In [11], certain terms in (20) (referred to as $Z_{B}$ terms) were incorrectly dropped. This was corrected in [12], which led to the expression in the last section for the kernel $G$.)

The primary difference of the two works [11, 12] is the choice of $\digamma_{0}$. The choice made in [12] was better suited to the diagrammatic expansion, and the resulting integral equation effectively sums up an infinite number of diagrams. However it was found for the present problem that this integral equation only has solutions in a very limited range of temperature and chemical potential, indicating that the sum of diagrams does not converge. In contrast, it turns out the choice of $\digamma_{0}$ made in [11] does not suffer from this problem. The latter $\digamma_{0}$ also has an appealing physical interpretation, as we now explain. Consider

$$
\digamma_{0}=\int \frac{d^{2} \mathbf{k}}{(2 \pi)^{2}}\left(\left(\omega_{\mathbf{k}}-\mu\right) f-\frac{1}{\beta}[(f-1) \log (1-f)-f \log f]\right)
$$


where $\omega_{\mathbf{k}}$ is the 1-particle energy of the free theory. The above expression can be interpreted as $\digamma_{0}=\epsilon-T s$, where $\epsilon$ is the first $(\omega-\mu) f$ term and represents the energy density. The remaining term represents the entropy density $s[24]$. This choice of $\digamma_{0}$ also more closely parallels the derivation of the thermodynamic Bethe ansatz[13].

Let us parameterize the occupation numbers in terms of a pseudo-energy $\varepsilon$ :

$$
f(\mathbf{k})=\frac{1}{e^{\beta \varepsilon(\mathbf{k})}+1}
$$

Then the variational equation $\delta \digamma / \delta f=0$ can be expressed in the simpler form:

$$
\varepsilon(\mathbf{k})=t \omega_{\mathbf{k}}-\mu-t \int \frac{d^{2} \mathbf{k}^{\prime}}{(2 \pi)^{2}} G\left(\mathbf{k}, \mathbf{k}^{\prime}\right) \frac{1}{e^{\beta \varepsilon\left(\mathbf{k}^{\prime}\right)}+1}
$$

(We have restored the hopping coupling $t$ here.) Using the above equation in $\digamma$, the free energy density $\mathcal{F}$ can be expressed as

$$
\mathcal{F}=-T \int \frac{d^{2} \mathbf{k}}{(2 \pi)^{2}}\left[\log \left(1+e^{-\beta \varepsilon}\right)+\frac{\beta}{2} \frac{1}{e^{\beta \varepsilon}+1}\left(\varepsilon-\omega_{\mathbf{k}}+\mu\right)\right]
$$

Comparing with [12], one sees that in the limit of small $G$, the equations presented there reduce to eqns. (26]27).

For two-component fermions, the occupation numbers are parameterized in terms of two pseudo-energies $\varepsilon_{\uparrow, \downarrow}$, and they satisfy a coupled system of two integral equations:

$$
\varepsilon_{\uparrow}(\mathbf{k})=t \omega_{\mathbf{k}}-\mu_{\uparrow}-t \int \frac{d^{2} \mathbf{k}^{\prime}}{(2 \pi)^{2}} G\left(\mathbf{k}, \mathbf{k}^{\prime}\right) \frac{1}{e^{\beta \varepsilon_{\downarrow}\left(\mathbf{k}^{\prime}\right)}+1}
$$

and the same equation with $\uparrow \leftrightarrow \downarrow$. Here the kernel $G$ is related to the scattering of spin up with spin down particles. By the $\mathrm{SU}(2)$ symmetry, for equal chemical potentials $\mu_{\uparrow}=\mu_{\downarrow} \equiv \mu, \varepsilon_{\uparrow}=\varepsilon_{\downarrow} \equiv \varepsilon$, and one only needs to solve one integral equation. The occupation number for each spin component has the form of a free theory given in (25), and the total density is 2 times the expression in (21), as is the free energy. 
For the Hubbard model, since we scaled out $t$ and the lattice spacing, everything then depends on the dimensionless variables $\widehat{\mu} \equiv \mu / t, \widehat{T} \equiv T / t, \widehat{t^{\prime}}=t^{\prime} / t$. Note that all the temperature dependence is in $\widehat{T}$, thus possible phase transitions should occur at fixed values of $T / t$, for given $g, t^{\prime}$. Henceforth we drop the hats, it being implicit that $T, \mu$ and $t^{\prime}$ are in units of $t$. The free energy density then takes the form:

$$
\mathcal{F}=-\frac{T}{a^{2}} c(\mu, T)
$$

where we have defined a scaling function $c$ (we suppressed the dependence on $t^{\prime}$ ):

$$
c=2 \int \frac{d^{2} \mathbf{k}}{(2 \pi)^{2}}\left[\log \left(1+e^{-\beta \varepsilon(\mathbf{k})}\right)+\frac{\beta}{2} \frac{1}{e^{\beta \varepsilon}+1}\left(\varepsilon(\mathbf{k})-\omega_{\mathbf{k}}+\mu\right)\right]
$$

It will also be convenient to express the density $n=-\partial \mathcal{F} / \partial \mu$ as

$$
n=\frac{2 q(\mu, T)}{a^{2}}=\frac{1-h}{a^{2}}
$$

where

$$
q=\int \frac{d^{2} \mathbf{k}}{(2 \pi)^{2}} \frac{1}{e^{\beta \varepsilon(\mathbf{k})}+1}
$$

Since $2 q$ is the number of particles of either spin per lattice site, half-filling corresponds to $q=1 / 2$. The quantity $h$ then corresponds to hole doping when it is positive, otherwise it represents particle doping. More precisely, $h$ is the number of holes per plaquette and the lattice is completely depopulated at $h=1$.

In order to probe the properties of the model, we will need a few other thermodynamic quantities. As usual the pressure $p=-\mathcal{F}$. Consider first the entropy per particle, $S / N=s / n$, where the entropy density $s=-\partial \mathcal{F} / \partial T$. It can be expressed in terms of the scaling functions as follows:

$$
\frac{S}{N}=\frac{1}{2 q}\left(c+T \partial_{T} c\right)
$$

The energy density $\epsilon=E / V=T s+\mu n+\mathcal{F}$. Thus the energy per particle is

$$
\frac{E}{N t}=\frac{\epsilon}{n t}=\mu+\frac{T^{2}}{2 q} c
$$


The specific heat per particle $C_{V} / N$ at constant volume and particle number $N$ is slightly more complicated since one must impose the constant density constraint. Setting $d q / d T=0$ relates $\mu$ and $T$ derivatives as follows:

$$
\frac{\partial T}{\partial \mu}=-\frac{\partial_{\mu} q}{\partial_{T} q}
$$

Using this, the specific heat per particle has the following expression:

$$
\frac{C_{V}}{N}=\frac{1}{N}\left(\frac{\partial E}{\partial T}\right)_{N, V}=\frac{T}{q}\left(\partial_{T} c+\frac{T}{2} \partial_{T}^{2} c-\frac{\left(\partial_{T} q\right)^{2}}{\partial_{\mu} q}\right)
$$

\section{THE FREE ENERGY AND ESTIMATES OF CRITICAL TEMPERA- TURES}

In this section we analyze the thermodynamics based on the formulas of the last section, provide evidence for instabilities, which may perhaps be phase transitions, and estimate critical temperatures.

In order to study the free energy, one must first solve the integral equation (26) for the pseudo-energy $\varepsilon$. This can be done iteratively, i.e. one starts with the approximation $\varepsilon_{0}=\omega_{\mathbf{k}}-\mu$ and plugs this into the right hand side to generate $\varepsilon_{1}$; this procedure is repeated until the solution converges. We approximated the integral equation by approximating the Brillouin zone as a $10 \times 10$ grid, and performing the integrals as discrete sums. This is rather crude, and was due to our limited computing resources; certainly one can do better. It was found that for large portions of the $\mu, T$ parameter space, the iterative procedure converged rapidly, typically within 10 iterations.

For reasons stated above, our analysis was performed for $g=15$, and $t^{\prime} / t=-0.3$. For fixed doping $h$, the chemical potential depends on temperature, but we find this dependence to be weak. In Figure 7 we plot hole doping $h$ as a function of chemical potential at the low temperature $T=0.2$, and it is nearly a straight line. 
As expected, increased doping corresponds to decreasing chemical potential; half filling occurs around $\mu=0.5$. This positive value of $\mu$ at half-filling is due to the mainly repulsive interactions.

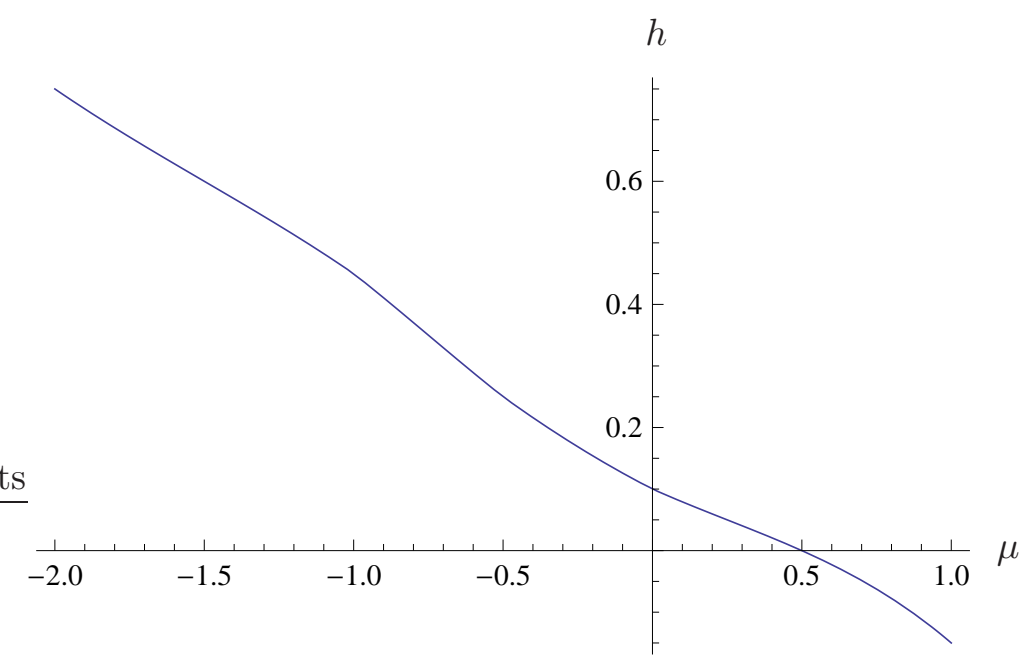

FIG. 7: Hole doping $h$ as a function of chemical potential for $T=0.2$.

The computed Fermi surfaces for various hole doping are shown in Figure 6. In this figure they are defined as the contour where the filling fraction $f=1 / 2$ at the low temperature $T=0.025$. They are in good agreement with experiments[22], especially in the anti-nodal directions, whereas in the nodal directions they are pulled back toward the center in a more pronounced manner in the data. This can likely be accounted for by adding additional hopping terms, such as next-to-next nearest neighbor.

The most interesting feature of the integral equation (26) is that there are regions in $\mu, T$ where the iterative procedure does not lead to a solution for an arbitarily high number of iterations; the procedure leads to $\varepsilon$ that jumps successively between two values, neither of which are solutions. (Figure 8.) Let us assume that in these regions, no solution exists, although our observations do not necessarily prove this. 
Furthermore, let us adopt the following physical interpretation. By comparison, in the standard BCS theory of superconductivity, one has a finite temperature gap equation. As the temperature is raised, at the critical temperature there are no longer solutions to this gap equation; i.e. as far temperature is concerned, it is a bottom up approach. In contrast, the present formalism is a top down approach: as the temperature is lowered one reaches a critical temperature where solutions no longer exist. Let us interpret this as an instability toward formation of a new phase, or perhaps a cross-over to a different behaviour. In support of this interpretation, we mention the treatment of the unitary Bose gas within the present formalism[14]. The gas undergoes a phase transition to a Bose-Einstein condensate at a critical value of $\mu / T$; above this value there are no solutions to the pseudo-energy integral equation.

It should be emphasized that the true nature of this 'phase' cannot be surmised from our thermodynamic approach alone; in addition one needs a bottom up approach that contains information about the zero temperature ground state, such as a gap equation. Such a complementary bottom up approach is developed in [28], where solutions to a BCS-like gap equation based on the attractive interactions described in section III are studied. The solutions are highly anisotropic, in that they vanish in the nodal directions, and are largest in the anti-nodal, and the critical $T_{c} \approx 0.04$ found there for $h=0.15$ is consistent with the critical temperatures estimated below.

Figure 8 indicates the regions where solutions do not exist for positive hole doping $h<0.25$. This figure is a contour plot of an interpolating function defined to be equal to 1 if there is a solution, and zero otherwise; the white region indicates the region of no solution, whereas in the light blue region, solutions exist. The boundary between the regions of existence and non-existence of solutions are the darkest curves, which are reasonably well delineated. Due to the 2-body approximation we have made in the thermodynamics, this boundary is not to be taken as precisely determined. The roughness of the boundaries we believe is a numerical artifact, mainly attributed to 
not using a fine enough grid in the temperature and chemical potential variables. The dip around $h=0.15$ we also believe to be an artifact since it disappears upon varying $g$ and $t^{\prime}$. In the range of doping $0.03<h<0.2$ one sees a possible phase transition with critical temperatures ranging from $0<T_{c}<.05$. As explained in Section III, since this is the range of doping where the Fermi surface is intersecting the attractive band in the anti-nodal directions, we propose that this signifies an instability toward the formation of Cooper pairs, so that superconductivity may occur in the white regions. At hole doping $h=0.15, T_{c} \approx 0.02$. This is reasonable, since experimentally $T_{c, \max } / t \approx 0.025$.

The single quasi-particle energies correspond to $\varepsilon(\mathbf{k})+\mu$. In Figure 9 we plot this single particle energy as a function of temperature at optimal hole doping $h=0.15$ in the anti-nodal direction. One clearly sees a drop at $T_{c, \max }$.

The higher $T_{c}$ 's up to 0.05 in the strongly underdoped region possibly signify the so-called pseudogap scale $T^{*}$. There appears to be a small separation around $h=.08$, however this is less pronounced for other $g$, so it is not clear if this signifies anything. Some recent experimental results are very relevant to the issue [25, 26]. Remarkably, it was found that the superconducting gap smoothly evolves into the pseudogap, i.e. they both seem to arise from the same underlying mechanism. In other words the gap is physically present even in regions of no superconductivity. This suggests that the $T_{c}$ 's in Figure 8 may all be arising from the same underlying phenomenon. This is consistent with the complimentary gap equation analysis in [28], where it was found that the gap extends and increases all the way to zero doping, as does the critical temperature scale in Figure 8. Although not shown in Figure 8, at higher doping there is another region of no-solutions with a maximum $T / t \approx 0.07$. This could perhaps signify the temperature referred to as $T_{\text {coh }}$ in the literature, where a crossover in the resistivity is observed from $\rho \propto T$ to $\rho \propto T+T^{2}$ [27].

We turn next to the thermodynamic functions, such as energy and entropy per 


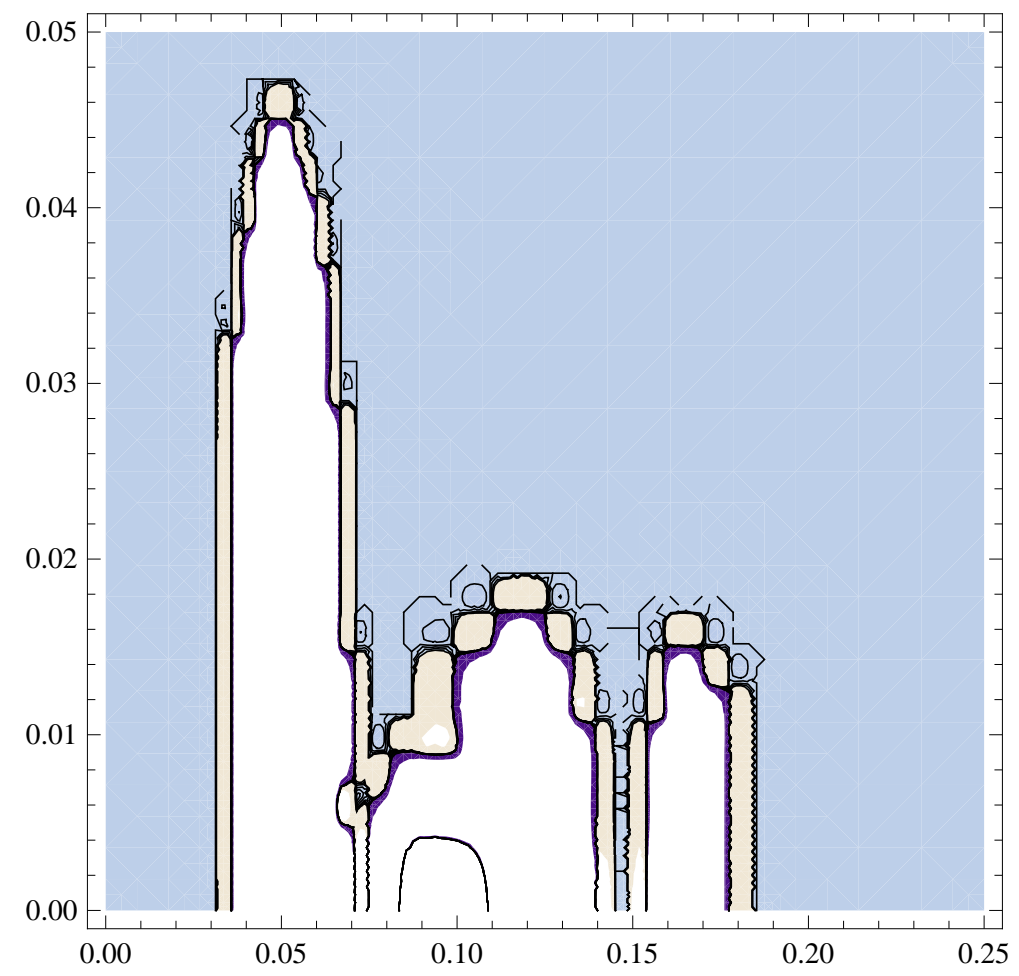

FIG. 8: Existence of solutions based on the iterative method. In the light regions there are no solutions to the integral equation for the pseudo-energy. The horizontal axis is the hole doping $h$, and the vertical axis is the temperature $T$. (See text for a detailed explanation.) particle. For hole densities in the vicinity of the boundaries shown in Figure 8, our crude solution to the integral equation for the pseudo-energy is not smooth enough to reliably compute temperature derivatives numerically. However, at low density our numerical results are better behaved, and although of less interest physically for the cuprates, at least allow a comparison with previous literature. We therefore analyzed the thermodynamics in the overdoped region, with $h=0.8$. The Fermi surface is shown in Figure 10, In Figures 11, 12 and 13, we plot the energy and entropy per particle and specific heat as a function of temperature. Our results 


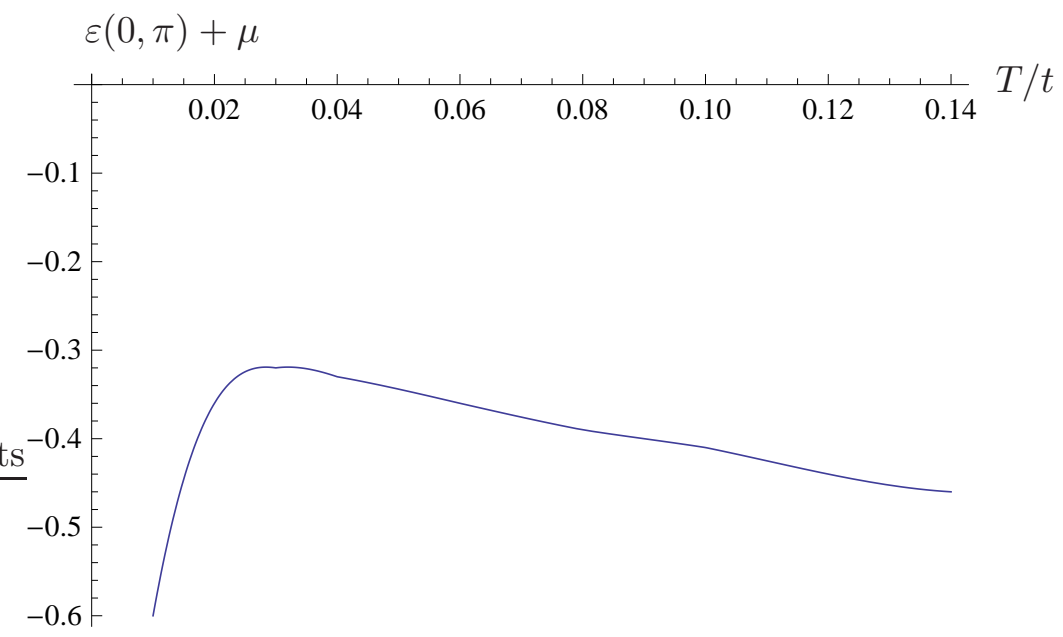

FIG. 9: The single particle energy $\varepsilon(\mathbf{k})+\mu$ in the anti-nodal direction $\mathbf{k}=(0, \pi)$ at optimal hole doping $h=0.15$ as a function of temperature.

for the entropy and specific heat are roughly consistent with the results in [8, 9], especially the results in [8], which extend to low density; a detailed comparison is beyond reach since previous results are typically at higher temperatures. 


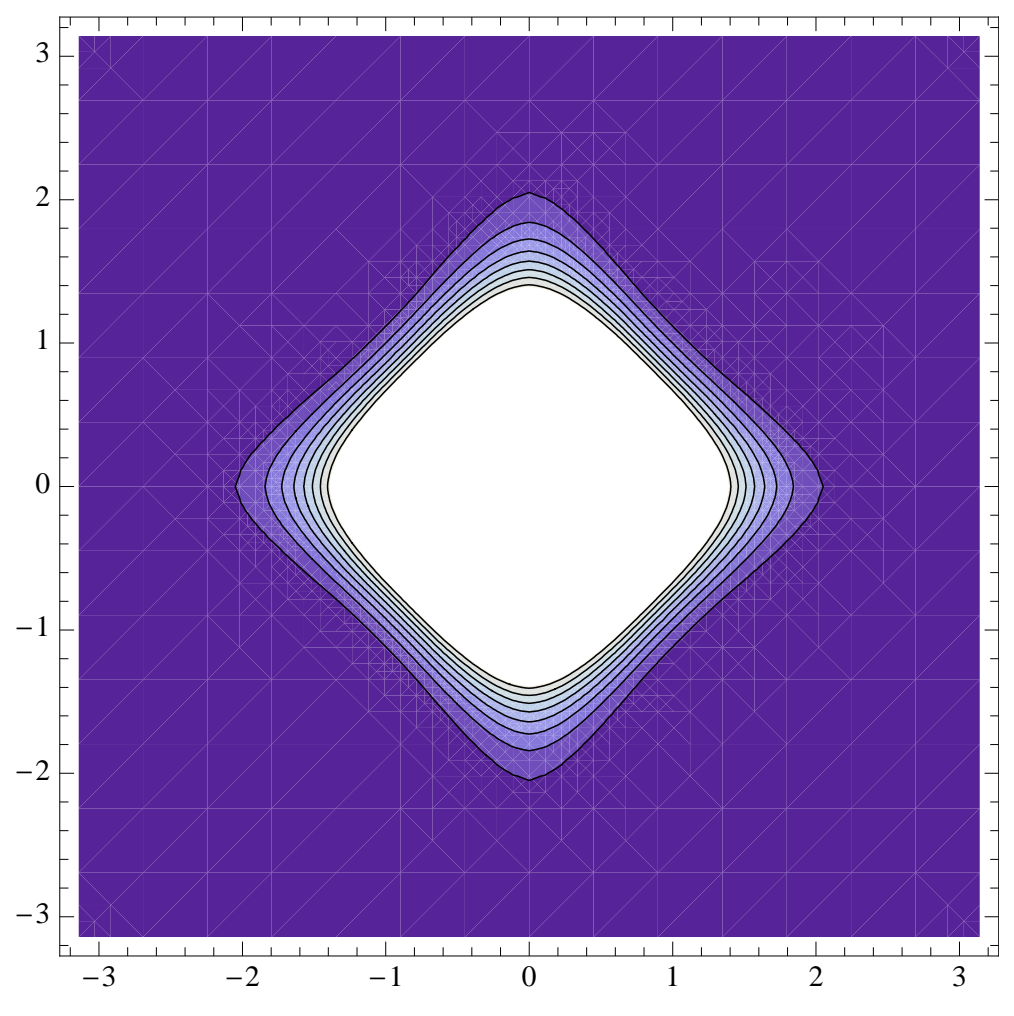

FIG. 10: The Fermi surface for hole doping $h=0.8$ at temperature $T=0.2$. The white region has $f=1$ and the darkest (purple) region $f=0$.

\section{CONCLUSIONS}

We presented an analytic treatment of the two-dimensional Hubbard model at finite chemical potential and temperature based on a new approach to statistical mechanics we recently developed[11, 12], which is built upon the S-matrix. The effective momentum-dependent coupling in this approach is the kernel $G$ of an integral equation satisfied by the pseudo-energy $\varepsilon$, which is built on the exact two-body Smatrix. We showed that there are regions of the Brillouin zone where the interactions are effectively attractive, for example, $U / t>6.4$ for $t^{\prime} / t=-0.3$, even though the 


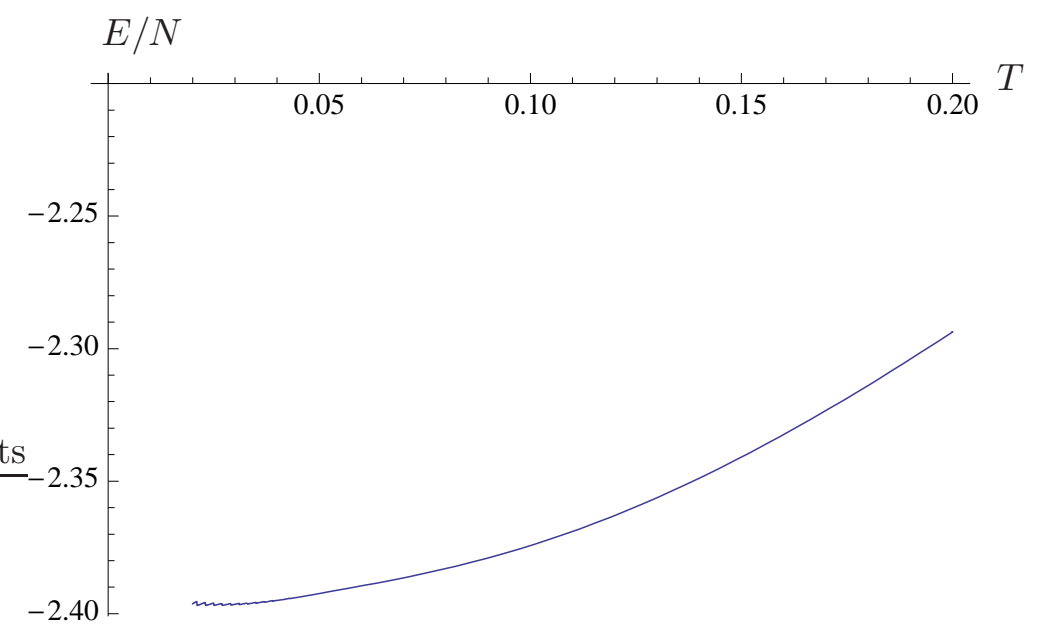

FIG. 11: The energy per particle as a function of temperature for hole doping $h=0.8$.

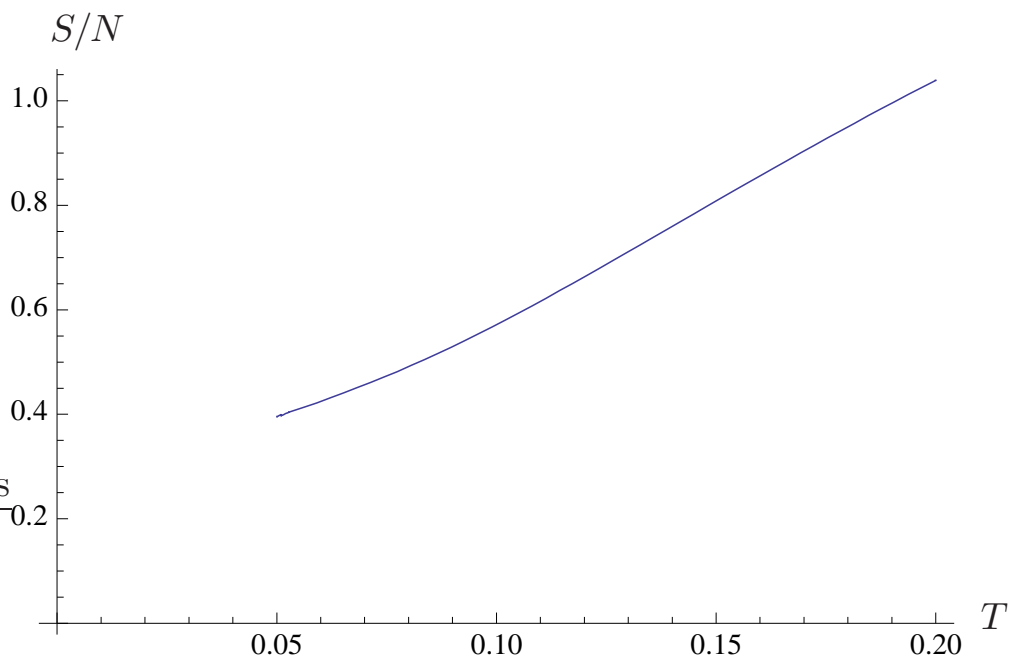

FIG. 12: Entropy per particle as a function of temperature for hole doping $h=0.8$. bare model only has repulsive interactions, and this is essentially due to multi-loop quantum corrections. The next-to-nearest neighbor hopping coupling $t^{\prime}$ plays a significant role in determining this property, and our analysis suggests that for a fixed value of $U / t$, superconductivity may not exist for $t^{\prime}=0$. We emphasize that no ap- 


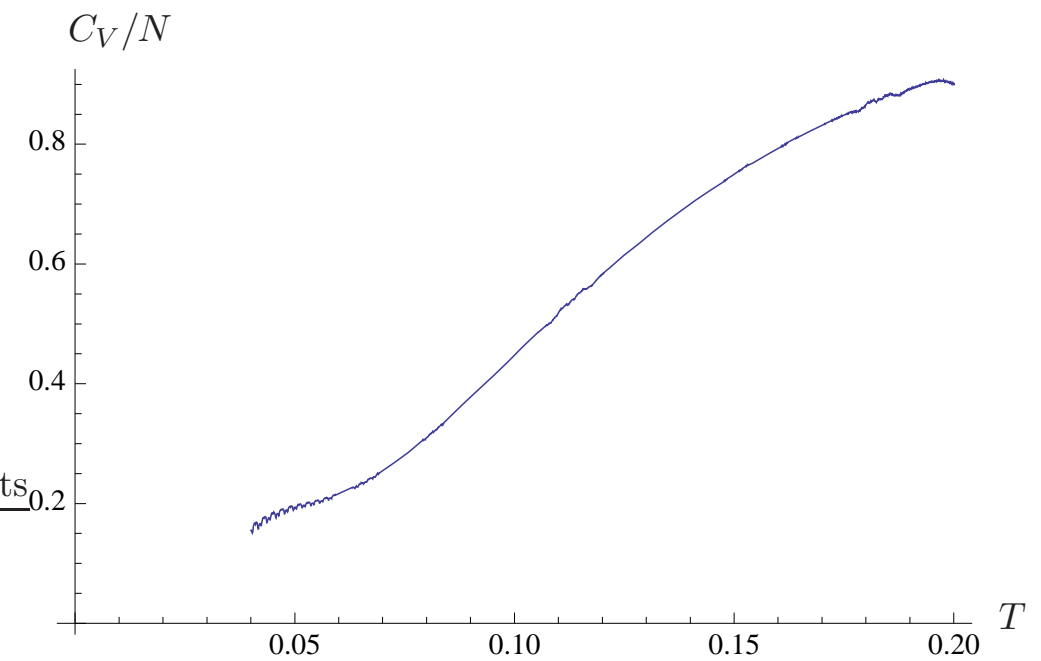

FIG. 13: Specific heat per particle as a function of temperature for hole doping $h=0.8$. proximations were made in obtaining these results; e.g. the existence of a threshold in $U / t$ for the existence of certain attractive interactions, stands on its own and is independent of the subsequent approximations we made in the thermodynamics.

We postulated that phase transitions occur where there are no solutions of the integral equation for the pseudo-energy. On the overdoped side, this phase sets in at hole doping $h<0.25$. Our result for $T_{c} \approx 0.02$ at $h=0.15$ is in good agreement with experiments. We found that there is also evidence for transitions in the underdoped region, and we suggested this signifies the pseudogap.

The main lesson of this work is that quantum loop corrections to scattering are perhaps the origin of the attractive interactions that lead to Cooper pairing near the Fermi surface. If this idea is correct, then in order to complete the picture one needs to derive a gap-equation that describes the structure of the ground state at zero temperature. Some preliminary attempts in this direction were taken in [28], where solutions to a gap equation based on the attractive interactions described above are studied, and the critical temperatures found are consistent with the bottom down 
approach described in this paper. Based on the detailed properties of the solutions to the gap equation studied there, i.e. its anisotropy and the existence of Fermi arcs in the nodal direction, it was suggested that the attractive mechanism in this paper may be responsible for the pseudogap rather than d-wave superconductivity.

\section{ACKNOWLEDGMENTS}

I would like to thank Henry Tye for discussions and Eliot Kapit and Kyle Shen for their help in understanding the phenomenology of high $T_{c}$ superconductivity. This work is supported by the National Science Foundation under grant number NSF-PHY-0757868.

[1] P. W. Anderson, The resonating valence bond state in $\mathrm{La}_{2} \mathrm{CuO}_{4}$ and superconduction, Science 235 (1987) 1196.

[2] D. Hone and P. Pincus, High temperature properties of the half-filled-band Hubbard model, Phys. Rev. B7 (1973) 4889.

[3] J. Hirsch, Two dimensional Hubbard model: Numerical simulation study, Phys. Rev. B31 (1985) 4403.

[4] E. Dagotto, Rev. Mod. Phys. 66 (1994) 763.

[5] A. Georges, G. Kotliar, W. Krauth and M. J. Rozenberg, Dynamical mean-field theory of strongly correlated fermion systems and the limit of infinite dimensions, Rev. Mod. Phys. 68 (1996) 13.

[6] P. van Dongen, Phase diagram of the extended Hubbard model at weak coupling, Phys. Rev. B54 (1996) 1584.

[7] M. Potthoff, Two-site dynamical mean field theory, Phys. Rev. B64 (2001) 165114. 
[8] J. Bonca and P. Prelovsek, Thermodynamics of the planar Hubbard model, Phys. Rev. B67 (2003) 085103.

[9] G. Li, W. Hanke and A. N. Rubstov, Accessing thermodynamics from dynamical cluster-embedding approaches, Phys. Rev. B80 (2009) 195118.

[10] C. N. Varney, C.-R. Lee, Z. J. Bai, S. Chiesa, M. Jarrell and R. T. Scalettar, Quantum Monte Carlo study of the two-dimensional fermion Hubbard Model, Phys. Rev. B80 (2009) 075116 [arXiv:0903.2519].

[11] A. LeClair, Quantum statistical mechanics of gases in terms of dynamical filling fractions and scattering amplitudes, J. Phys. A40 (2007) 9655 arXiv:hep-th/0611187.

[12] P.-T. How and A. LeClair, Critical point of the two-dimensional Bose gas: an S-matrix approach, Nucl. Phys. B824 (2010) 415 arXiv:0906.0333.

[13] C. N. Yang and C. P. Yang, Jour. Math. Phys. 10, (1969) 1115.

[14] P.-T. How and A. LeClair, S-matrix approach to quantum gases in the unitary limit I: the two-dimensional case, J. Stat. Mech. (2010) P03025 arXiv:1001.1121]; S-matrix approach to quantum gases in the unitary limit II: the three-dimensional case, J. Stat. Mech. (2010) P07001 arXiv:1004.5390.

[15] A. LeClair On the viscosity to entropy density ratio for unitary Bose and Fermi Gases, New J. Phys. 13 (2011) 055015 [arXiv: 1012.5653].

[16] F. H. L. Essler and V. E. Korepin, Complete Solution of the one-dimensional Hubbard Model, Phys. Rev. Lett 67 (1991) 3848.

[17] H. Kondo and T. Moriya, Superconductivity in 2D Organic Compounds and High $T_{c}$ Cuprates, J. Phys. Soc. Jpn, 68 (1999).

[18] S. Raghu, S. A. Kivelson and D. J. Scalapino, Superconductivity in the repulsive Hubbard model: An asymptotically exact weak-coupling solution, Phys. Rev. B81 (2010) 224505 .

[19] L. Balents and M. P. A. Fisher, Weak Coupling Phase Diagram of the Two Chain 
Hubbard Model, Phys. Rev. B53 (1996) 12133 arXiv:cond-mat/9503045].

[20] L. Simonelli et. al, The Material-Dependent Parameter Controlling the Universal Phase Diagram of the Cuprates, Journal of Superconductivity: Incorporating Novel Magnetism 18 (2005) 773.

[21] L. N. Cooper, Bound Electron pairs in a Degenerate Fermi Gas, Phys Rev. 104 (1956) 1189.

[22] H. Ding, J. C. Campuzano, A. F. Bellman, T. Yokoya, M. R. Norman, M. Randeria, T. Takahashi, H. Katayama-Yoshida, T. Mochiku, K. Kadowaki, and G. Jennings, Momentum Dependence of the Superconducting Gap in $\mathrm{Bi}_{2} \mathrm{Sr}_{2} \mathrm{CaCu}_{2} \mathrm{O}_{8}$. Phys. Rev. Lett. 74 (1995) 2784.

[23] R. Dashen, S.-K. Ma, and H. J. Bernstein, S-matrix Formulation of Statistical Mechanics, Phys. Rev. 187 (1969) 345.

[24] L. D. Landau and E. M. Lifshitz, Statistical Physics, Pergamon Press (1980).

[25] Y. Kohsaka, C. Taylor, P. Wahl, A. Schmidt, Jhinhwan Lee, K. Fujita, J. Alldredge, Jinho Lee, K. McElroy, H. Eisaki, S. Uchida, D.-H. Lee, and J.C. Davis, How Cooper pairs vanish approaching the Mott insulator in $\mathrm{Bi}_{2} \mathrm{Sr}_{2} \mathrm{CaCu}_{2} \mathrm{O}_{8+\delta}$. Nature 454 (2008) 1072 .

[26] U. Chatterjee et. al. Observation of a d-wave nodal liquid in highly underdoped $\mathrm{Bi}_{2} \mathrm{Sr}_{2} \mathrm{CaCu}_{2} \mathrm{O}_{8+\delta}$, Nature Phys. 6 (2010) 99 [arXiv:0910.1648].

[27] N. E. Hussey, Phenomenology of the normal state in-plane transport properties of high-T $T_{c}$ cuprates, J. Phys: Cond. Matter 20 (2008) 123201 arXiv:0804.2984.

[28] A. LeClair, Superconductivity in the two-dimensional Hubbard model based on the exact pair potential, arXiv:1008.5116. 